\title{
\begin{tabular}{l|l} 
pcori $).$ & PATIENT-CENTERED OUTCOMES RESEARCH INSTITUTE \\
RESEARCH SUMMARY
\end{tabular}
}

\section{Follow-Up Care Strategies after Treatment for Breast Cancer}

Principal investigator

Caprice C. Greenberg, MD, MPH
Organization

The Alliance for Clinical Trials in Oncology Foundation

\section{What was the research about?}

After women complete treatment for breast cancer, they may still be at risk for cancer to come back. But not all breast cancer is the same. For example, some breast cancers have hormone receptors. Cancers that have these receptors depend on hormones, like estrogen and progesterone, to grow. Breast cancers can also have a high level of a protein called HER2. Doctors call these cancers HER2+. These cancers grow faster than other cancers.

Women and their doctors watch for signs of cancer coming back. To check for these signs, women usually get regular mammograms. In addition to regular mammograms, doctors may recommend extra imaging tests. Some doctors recommend extra imaging tests even when patients feel well. Others only recommend additional imaging tests if their patients have physical signs of cancer coming back.

The research team did two studies. In the first study, the team wanted to know if there was a link between different types of breast cancer and the chance that cancer will come back, and how long it takes to come back. In the second study, the team looked at the extra imaging tests many women receive. They wanted to know if women without physical signs of cancer who got extra imaging tests had a higher chance of survival from cancer that came back within five years. The team compared these women to women who got extra imaging tests only if they had physical signs of cancer.

\section{What were the results?}

Study 1. Cancer came back sooner and more often for women whose cancer didn't have hormone receptors and also wasn't HER2+ compared with women whose cancer had other combinations of hormone receptors and HER2.

Study 2. Compared with women who received extra imaging only if they had physical signs of cancer, two groups of women who received extra imaging without physical signs of cancer had higher chances of survival:

- Women with cancer that didn't have either type of hormone receptors and wasn't HER2+

- Women with cancer that was HER2+

For women with cancer that was not HER2+ but had hormone receptors, there was no difference in survival based on which way they received follow-up imaging tests.

\section{What did the research team do?}

In the first study, the research team analyzed data from 17 research studies to see if hormone receptors and the HER2 protein affected the chance that breast cancer would come back. The studies included 10,357 women with breast cancer.

In the second study, the research team analyzed data from 10,076 women to see if women who had extra imaging tests without physical signs of cancer had higher chances of survival from cancer that came back. The team compared these women to women 
who only had extra imaging tests if they had physical signs of cancer.

A group of 31 cancer survivors, patient advocates, and cancer specialists helped design both studies.

\section{What were the limits of the study?}

The research team didn't have information about other risk factors, such as family history of breast cancer. These risk factors may be important when deciding how to look for signs of breast cancer that comes back.
Future research could assign patients by chance to get extra imaging tests or not. These studies could help make sure that if there are differences in survival, the differences are due to how women get extra imaging tests and not due to other things.

\section{How can people use the results?}

Patients and doctors can use the results from this study to help plan follow-up care after breast cancer treatment.

To learn more about this project, visit www.pcori.org/Greenberg177. 